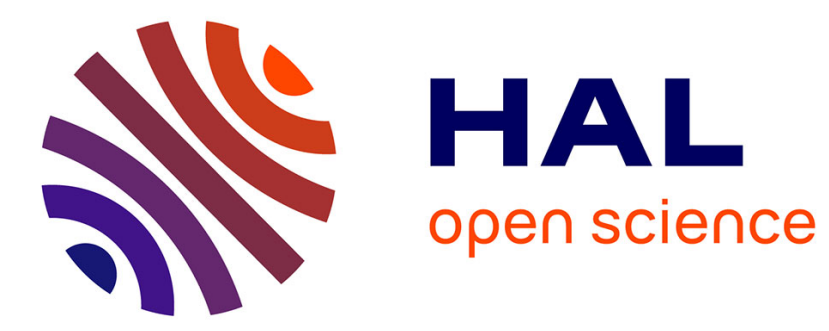

\title{
Optimal light harvesting in 2D semiconductor heterostructures
}

Zhesheng Chen, Johan Biscaras, Abhay Shukla

\section{To cite this version:}

Zhesheng Chen, Johan Biscaras, Abhay Shukla. Optimal light harvesting in 2D semiconductor heterostructures. 2D Materials, 2017, 4, pp.025115 10.1088/2053-1583/aa736f . hal-01552781

\section{HAL Id: hal-01552781 \\ https://hal.sorbonne-universite.fr/hal-01552781}

Submitted on 3 Jul 2017

HAL is a multi-disciplinary open access archive for the deposit and dissemination of scientific research documents, whether they are published or not. The documents may come from teaching and research institutions in France or abroad, or from public or private research centers.
L'archive ouverte pluridisciplinaire HAL, est destinée au dépôt et à la diffusion de documents scientifiques de niveau recherche, publiés ou non, émanant des établissements d'enseignement et de recherche français ou étrangers, des laboratoires publics ou privés. 


\title{
Optimal light harvesting in 2D semiconductor heterostructures
}

\author{
Zhesheng Chen ${ }^{1,2,3}$, Johan Biscaras ${ }^{1}$, and Abhay Shukla ${ }^{1}$
}

1 Institut Institut de Minéralogie, de Physique des Matériaux et de Cosmochimie, Sorbonne Universités - UPMC Univ Paris 06, CNRSUMR7590, 4 Place Jussieu, Paris 75252, France

2 Laboratoire des Solides Irradiés, Ecole polytechnique, Université Paris Saclay, 91128 Palaiseau cedex, France

3 School of Nuclear Science and Technology, Lanzhou University, Lanzhou 730000, P.R. China

*Email: zheshengchen@gmail.com and abhay.shukla@upmc.fr

ABSTRACT: Optoelectronics with two dimensional (2D) heterostructures combining transition metal dichalcogenides (TMDCs) and other semiconductors in hybrid stacks is potentially promising because of the possibility of fabricating devices with high efficiency and new properties. Ultrafast charge transfer across the interface and long lifetime of carriers makes the vertical geometry attractive with respect to traditional bulk heterostructures. In such ultrathin structures, the multiple boundaries and the thickness of each material play a key role in the interaction of light with the device and can strongly influence the device performance. In this article we study light harvesting in 2D 
InSe/ $\mathrm{MoS}_{2}$ semiconductor heterostructures by measuring Raman enhancement or attenuation as a function of layer thicknesses. Measurements are precisely reproduced by the calculation of the light emission, and the field distribution inside the heterostructure. Optimizing layer thickness and material interfaces has a significant effect on the light distribution in such 2D heterostructures with layer thickness in the region of a few tens of nanometers, providing a means to enhance the performance of emerging 2D semiconductor-heterostructure optoelectronics.

KEYWORDS: 2D semiconductor heterostructure, $\mathrm{InSe} / \mathrm{MoS}_{2}$, Raman enhancement, light emission, light distribution

Van der Waals heterostructures made by stacking various 2D semiconductors onto each other (2D semiconductor-heterostructures) are of great interest because of the possibility of combining specific properties of each material in a device, which maintains a $2 \mathrm{D}$ character and can be ideally used in electronics and optoelectronics [1-11]. In comparison to a 'bulk' semiconductor-heterostructure with layer thickness of the order of the light wavelength or bigger, the multiple interfaces and the sub-wavelength thickness of each layer decisively influence the interaction of light with heterostructures, and the device performance. An example of combining specific properties is provided by an atomically thin device of ptype monolayer $\mathrm{MoS}_{2}$ and n-type monolayer $\mathrm{WSe}_{2}$ designed to benefit from the direct gaps in TMDCs monolayers [12]. This device provides unconventional performance with gate tunable diode-like current 
rectification combined with photovoltaic response across the $p-n$ interfaces. A thicker device made of $p$ type few-layer $\mathrm{MoS}_{2}$ and p-type few-layer $\mathrm{WS}_{2}$ has a rectifying and bipolar behavior and moreover can function not only as a photovoltaic cell but also as a self-driven photo-detector with photo-switching ratio exceeding $10^{3}$. This indicates strong light interaction in the device even though both few-layer TMDCs have indirect band gaps [13]. The performance of devices based on 2D heterostructures can also be improved by optimizing light absorption in the stack by coupling plasmonic nanoantennae or microcavities to the structures [14-16]. In 2D materials, there is another convenient way to enhance light coupling by modifying the layer structure and the dielectric surroundings of the device with a suitable substrate [17-22].

Raman spectroscopy is a widely used non-destructive characterization and investigation method in 2D materials with good energy and spatial resolution and mapping capability [23-26]. In non-resonant conditions it is also a reliable technique to study the interaction of light with 2D heterostructures, notably by measuring the enhancement or attenuation of Raman intensity with respect to that in isolated layers. Such an approach can be modeled by standard simulations and gives a new route to improve device performance. However in experimentally fabricated devices it is important to achieve very clean $2 \mathrm{D}$ semiconductor-heterostructures and interfaces since impurities and inhomogenities can critically influence the measured intensity. This may explain the fact that few reports focus on this approach for explaining and eventually predicting optimal device geometries.

In this work we fabricated different 2D semiconductor-heterostructures such as few layer InSe stacked on few layer $\mathrm{MoS}_{2}\left(\mathrm{InSe} / \mathrm{MoS}_{2}\right)$ by what we call a "random transfer" method. This is a fast, ultra-clean, 
high-yield technique. The Raman enhancement or attenuation factors in InSe/MoS with various thicknesses are directly observed when compared to the intensities of $\mathrm{InSe}$ and $\mathrm{MoS}_{2}$ vibrational modes separately, and also compared to a light scattering simulation which is in good agreement with our experiment results. One can thus design a 2D semiconductor-heterostructure by choosing the optimum thickness, substrate and stacking sequence to maximize device performance.

\section{RESULTS AND DISCUSSION}

The preferred method in the fabrication of clean 2D heterostructures is the dry transfer method, which includes the dual polymer transfer process and pick up technique. However, these techniques have the shortcoming that the surface of the sample might still have polymer residuals and that they are relatively time-consuming $[27,28]$. In our "random transfer" heterostructure fabrication process, we experimented direct mechanical exfoliation of one sample onto the other without water or polymer media (see details in Methods). The schematics of the sample-2/sample-1 heterostructure fabrication process are shown in

Figure 1 (a). Briefly, the bottom sample (sample 1) is firstly prepared by mechanical exfoliation on a substrate (glass or $\mathrm{SiO}_{2} / \mathrm{Si}$ ) on a hot plate. Considering the adhesion force of $2 \mathrm{D}$ precursors and the adhesive tape, the temperature of the hot plate is in the range $100{ }^{\circ} \mathrm{C}-125^{\circ} \mathrm{C}$ in our fabrication process. The comparison of adhesion of samples ( $\mathrm{MoS}_{2}$ and $\left.\mathrm{InSe}\right)$ fabricated under different temperatures is shown in the supplementary information 1. In the second step the top layer (sample 2) is then mechanically exfoliated on to the same zone on the heated substrate using the same procedure. After the second step one can find some overlapping areas on the substrate indicating the existence of pristine sample $1 /$ sample 2 heterostructures. There are several advantages of using this technique to fabricate heterostructures 
compared to other transfer methods. The whole process is very fast (within one minute) and no chemical or polymer residuals are found on the heterostructure. In addition the yield is very high as tens of heterostructures can be formed in one fabrication process as seen in supplementary information 2 . It is worth noticing that our method is based on random mechanical exfoliation, which means the thicknesses and the size of the layers of the heterostructure cannot be precisely controlled. However, it is very suitable for the study of properties based on a large amount of samples as in our case. All the heterostructures in this article are fabricated on $\mathrm{SiO}_{2}(285 \mathrm{~nm}) / \mathrm{Si}$ substrate except otherwise mentioned. The optical images and corresponding atomic force microscopy (AFM) of two typical 2D semiconductor-heterostructures such as InSe/MoS 2 and GaSe/InSe are shown in Figure 1(b-g). From the optical images, one can clearly see that the "color" of overlapping parts is different from different areas, already indicating the expected variation of the light interaction and absorption according to geometry. The corresponding AFM image of each heterostructure shows clean uniform surfaces, without bubbles or chemical residue that always appear during other transfer process. This is very critical for precise investigation of the interaction of light interaction with each element of the $2 \mathrm{D}$ heterostructure. The thicknesses of the random transferred 2D semiconductor-heterostructures shown in this study are InSe $(28.1 \mathrm{~nm}) / \mathrm{MoS}_{2}(20.2 \mathrm{~nm}), \mathrm{InSe}$ $(28.1 \mathrm{~nm}) / \mathrm{MoS}_{2}(33.9 \mathrm{~nm})$ and $\mathrm{GaSe}(19.1 \mathrm{~nm}) / \mathrm{InSe}(29.2 \mathrm{~nm})$ respectively. Since mechanical exfoliation method is widely used in the preparation of 2D materials our random transfer method is of relevance for laboratory $2 \mathrm{D}$ semiconductor-heterostructure fabrication. 

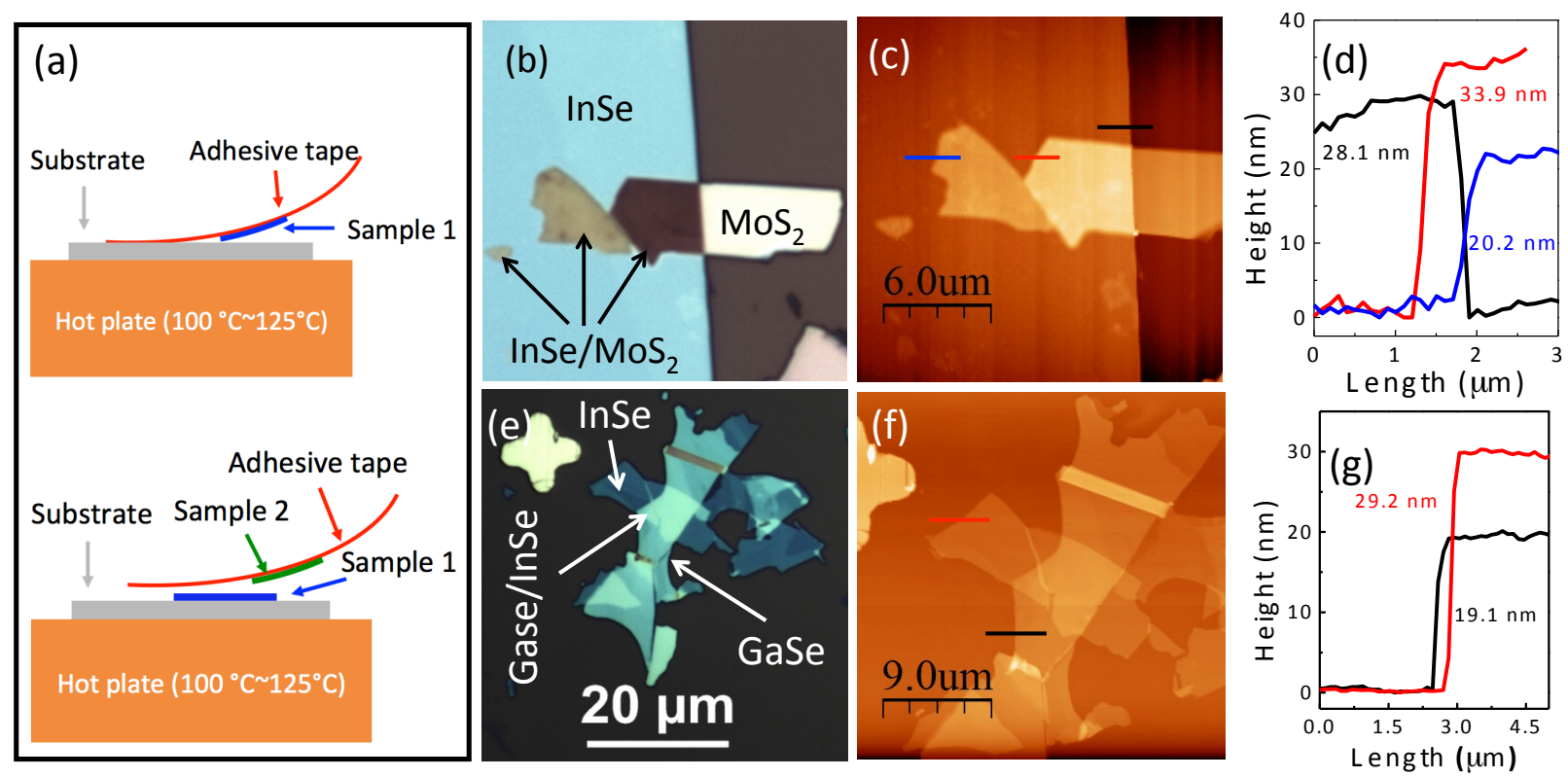

Figure 1. (a) The random transfer process for 2D semiconductor-heterostructure fabrication. (b) Optical image of InSe/ $\mathrm{MoS}_{2}$ heterostructure. (c-d) The corresponding AFM image of InSe/MoS $\mathrm{M}_{2}$ heterostructure and the height profiles of InSe and $\mathrm{MoS}_{2}$. (e) Optical image of GaSe/InSe heterostructure. (f-g) The corresponding AFM image of GaSe/InSe heterostructure and the height profiles of GaSe and InSe.

Measuring Raman scattering intensity is a direct way to investigate the interaction of light with $2 \mathrm{D}$ semiconductor-heterostructure. In our experiment, we use two different wavelengths to check the Raman intensity variation from each material. In non-resonant conditions, the Raman scattering intensity of an isolated layer should be proportional only to the scattering volume that is to layer thickness in the back scattering geometry. However for a layer of the same thickness of the same material in a heterostructure, this intensity will change depending on the interaction of light at the interfaces with other layers and with the substrate as well. As an illustration, consider the $\mathrm{InSe} / \mathrm{MoS}_{2}$ heterostructure of Figure 1(a). The Raman spectra with $532 \mathrm{~nm}$ excitation from the bare $\operatorname{InSe}(28.1 \mathrm{~nm})$ or $\operatorname{MoS}_{2}(33.9 \mathrm{~nm})$ layers on the substrate are shown in Figure 2(a) with black and blue lines. If now we compare the intensity from heterostructures, we find for example that the InSe $\mathrm{A}^{1}{ }_{1 \mathrm{~g}}$ mode intensity increases $\sim 3$ times in InSe $(28.1$ 
$\mathrm{nm}) / \mathrm{MoS}_{2}(33.9 \mathrm{~nm})$, while it decreases $\sim 2$ times in $\operatorname{InSe}(28.1 \mathrm{~nm}) / \mathrm{MoS}_{2}(20.2 \mathrm{~nm})$. This six-fold variation shows the importance of the thickness of the underlying $\mathrm{MoS}_{2}$ layer in regulating the lightmatter interaction in the InSe layer. In addition, the Raman intensity from $\mathrm{MoS}_{2}$ enhances with a six-fold increase in InSe $(28.1 \mathrm{~nm}) / \mathrm{MoS}_{2}(33.9 \mathrm{~nm})$. Finally we study the influence of the wavelength of the incident light by using the $638 \mathrm{~nm}$ laser for excitation. As before we note a dramatic variation of intensity. The $A^{1}{ }_{1 \mathrm{~g}}$ mode intensity of InSe decreases $\sim 20$ fold in InSe $(28.1 \mathrm{~nm}) / \mathrm{MoS}_{2}(33.9 \mathrm{~nm})$ and $\sim 40$ fold in InSe $(28.1 \mathrm{~nm}) / \mathrm{MoS}_{2}(20.2 \mathrm{~nm})$ respectively. We also note that this wavelength excites resonant scattering from $\mathrm{MoS}_{2}$ and as a result no deductions can be made concerning the $\mathrm{MoS}_{2}$ Raman lines nor the InSe $\mathrm{A}^{1}{ }_{2 \mathrm{~g}}$ mode at this wavelength [29]. The results above demonstrate the important role of the wavelength of incident light in the final scattered intensity. We then perform Raman mapping in this InSe/ $\mathrm{MoS}_{2}$ heterostructure to analyze the uniformity over the whole surface which is an important indication of the quality, cleanliness and homogeneity of the heterostructure. This also excludes the eventuality that the measured enhancements or attenuations could correspond to some singular spots or edge of the heterostructure. The Raman mapping area is that of Figure 1(b) and the data according to different vibration modes are shown in Figure 2(c), the border of the heterostructure being indicated by the dashed line. From the data, one can clearly see that the Raman intensity of each material in the different heterostructure areas is uniformly enhanced or attenuated as explained before. Moreover, it is worth noticing that the enhancement or attenuation of Raman intensity is also observed in other $2 \mathrm{D}$ semiconductor-heterostructures, such as GaSe/InSe heterostructure fabricated in Figure 1(e-f), the Raman intensity of both GaSe and InSe have significantly decreased in the heterostructure under both $532 \mathrm{~nm}$ 
and $638 \mathrm{~nm}$ excitation as shown in Figure 2(d-e). These changes in intensity can be attributed to the optical interactions within the heterostructure as we rule out mechanisms related to charge transfer or interlayer interactions as seen in some other heterostructures [30, 31].
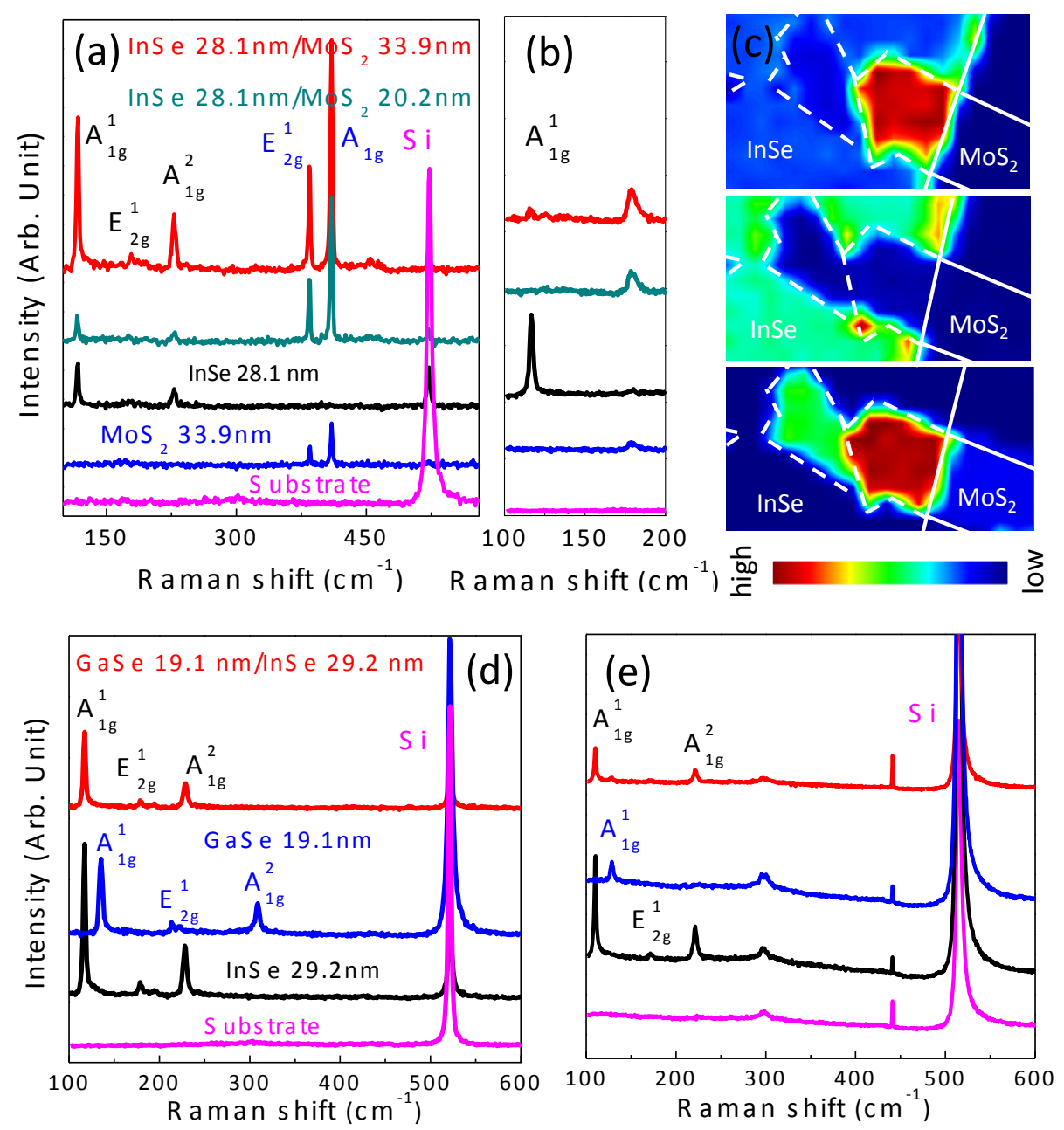

Figure 2. (a-b) Raman shift of InSe/MoS 2 heterostructure under $532 \mathrm{~nm}$ excitation and under $638 \mathrm{~nm}$ excitation respectively. (c) Raman mapping of $\mathrm{InSe} / \mathrm{MoS}_{2}$ heterostructure. From top to bottom: InSe $\mathrm{A}_{1 \mathrm{~g}}{ }^{1}$ mode under $532 \mathrm{~nm}$ excitation, InSe $\mathrm{A}_{1 \mathrm{~g}}^{1}$ mode under $638 \mathrm{~nm}$ excitation and $\mathrm{MoS}_{2} \mathrm{E}_{2 \mathrm{~g}}^{1}$ mode under 532 $\mathrm{nm}$ excitation. (d-e) Raman shift of GaSe/InSe heterostructure under $532 \mathrm{~nm}$ excitation and under $638 \mathrm{~nm}$ excitation respectively.

This strong enhancement or attenuation of the same Raman modes according to the wavelength used and the configuration of our $2 \mathrm{D}$ semiconductor-heterostructures can be explained by introducing a 
multiple reflection, normal incidence model, which has been successfully used to explain light outcoupling in 2D materials placed on a substrate [32-34]. However in our case we have to account for multiple layers of the heterostructure and calculate Raman intensity from each layer. A simple physical picture of the phenomenon is the following and the corresponding model is shown in the supplementary information 3: The factor multiplying the intensity of the Raman mode from the top layer (respectively the bottom layer) depends on two quantities: the incident wavelength field and the inelastically scattered wavelength field at a given depth $\mathrm{x}$ of the heterostructure. Considering the multiple interfaces in this $2 \mathrm{D}$ semiconductor-heterostructure, incident light will encounter the boundaries air/semiconductor-1, semiconductor-1/semiconductor-2, semiconductor- $2 / \mathrm{SiO}_{2}$ and $\mathrm{SiO}_{2} / \mathrm{Si}$, undergoing multiple reflections. Taking into account both the incident light at a given depth $\mathrm{x}$ in the layer and light emission from the same depth $\mathrm{x}$, the total intensity of light emission from the semiconductor-1 (semiconductor-2) layer can be defined as:

$$
I=A \int_{0}^{d}\left|E_{a b s}\left(x, \lambda_{1}\right) E_{e m i}\left(x, \lambda_{2}\right)\right|^{2} d x
$$

where $\mathrm{A}$ is a constant chosen to be 1 in our calculation, $\mathrm{d}$ is the thickness of semiconductor-1 (semiconductor-2) layer when we calculate the intensity of light emission from the top (bottom) semiconductor, $\lambda_{1}$ and $\lambda_{2}$ are the incident light wavelength and emitted light wavelength respectively, and $\mathrm{E}_{\mathrm{abs}}\left(\mathrm{x}, \lambda_{1}\right)$ and $\mathrm{E}_{\mathrm{emi}}\left(\mathrm{x}, \lambda_{2}\right)$ are the amplitude of the incident light field and the amplitude of the emitted field at depth $\mathrm{x}$ in the layer after taking into account interference effects due to reflection and transmission at the various interfaces. The model does not calculate the scattering process or the physical process for the generation of the emitted light, but simply takes into account the interactions with the various 
interfaces and layers both for the incident and emitted fields. Raman scattering is very weakly inelastic on this scale, for example, the scattered wavelength of the $A^{1}{ }_{1 \mathrm{~g}}$ mode of InSe is $535 \mathrm{~nm}\left(\lambda_{2}\right)$ under $532 \mathrm{~nm}$ excitation $\left(\lambda_{1}\right)$. In short a heterostructure which maximizes the emitted Raman intensity corresponding to a given layer will ensure maximum light-matter interaction in that layer and emission from the device. The inverse is also true. This thus permits the design of efficient heterostructures.

Let us first focus on the comparison of emission intensity between calculation and experiment from InSe in our InSe/ $\mathrm{MoS}_{2}$ heterostructures. Once the measured intensities have been normalized for incident power and measuring time, a universal normalizing factor is sufficient to compare data to calculation. In Figure 3(a) we compare the calculated excitation wavelength dependent emission intensity with the measured data for the excitation wavelength of $532 \mathrm{~nm}$ and $638 \mathrm{~nm}$ respectively (green and red stars). All the refractive indices used for $\mathrm{InSe}, \mathrm{MoS}_{2}, \mathrm{SiO}_{2}$ and $\mathrm{Si}$ were adopted from literatures [35-39], and are shown in Figure S5 of the supplementary information 3. Particularly, the refractive indices corresponding to $\mathrm{A}_{1 \mathrm{~g}}^{1}$ Raman mode of InSe and $\mathrm{E}_{2 \mathrm{~g}}^{1}$ Raman mode of $\mathrm{MoS}_{2}$ are listed in Table $\mathbf{S 1}$ of the supplementary information 3. It can be seen in both cases that our model accounts perfectly for the intensity changes in the different heterostructures with the change in the thickness of $\mathrm{MoS}_{2}$. Sharp features in curves between 600 and $700 \mathrm{~nm}$ wavelengths are due to the direct transitions in $\mathrm{MoS}_{2}$ which introduce sharp variations in the refractive index. In Figure 3(b) we examine the changes in emitted Raman intensity from $\mathrm{MoS}_{2}$ under different incident radiation, again with very good agreement between calculation and experimental data acquired under $532 \mathrm{~nm}$ excitation. In Figure 3(c-d) we show how the emission intensity varies with the thickness of a lower layer of $\mathrm{MoS}_{2}$ and the effect of the incident wavelength as an example of how to 
design an optimal InSe/MoS 2 heterostructure. In Figure 3(c) we fix the InSe thickness to $28.1 \mathrm{~nm}$ and calculate emitted intensity from $\mathrm{A}^{1}{ }_{1 \mathrm{~g}}$ Raman mode of InSe with respect to the variation of $\mathrm{MoS}_{2}$ layer thickness and the excitation wavelength. It can be seen that the optimal $\mathrm{MoS}_{2}$ thickness (maximizing Raman intensity from $\mathrm{InSe}$ ) is about $35 \mathrm{~nm}$ for an excitation wavelength of $532 \mathrm{~nm}$. However for an excitation wavelength of $638 \mathrm{~nm}$, a non-zero $\mathrm{MoS}_{2}$ layer actually decreases intensity. Finally in Figure 3(d) we calculate emitted intensity from the $\mathrm{E}_{2 \mathrm{~g}}^{1}$ Raman mode of $\mathrm{MoS}_{2}$ under the same conditions. The optimum $\mathrm{MoS}_{2}$ thickness is about $33 \mathrm{~nm}$ under $532 \mathrm{~nm}$ excitation.
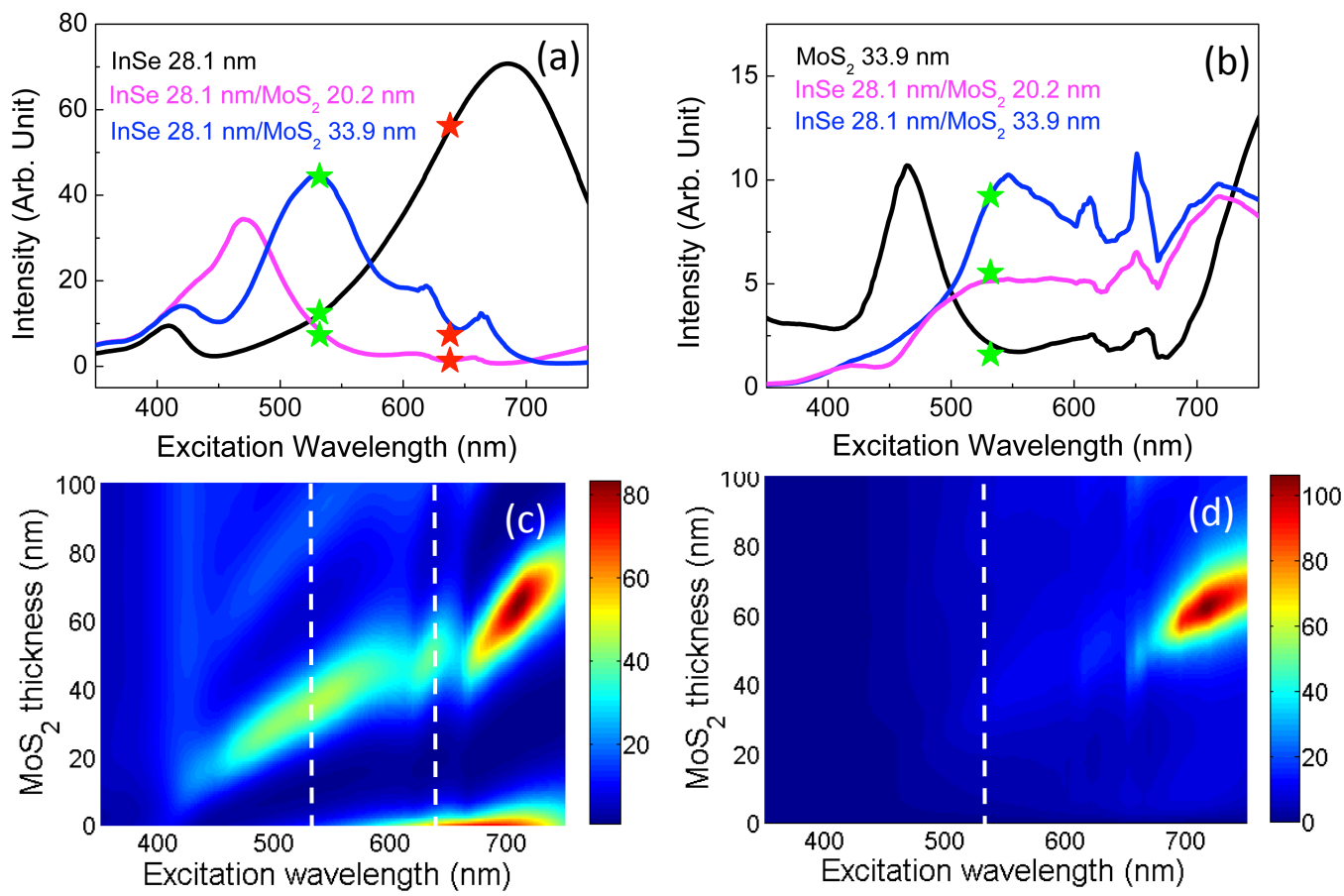

Figure 3. Light emission intensity of InSe $\mathrm{A}_{1 \mathrm{~g}}{ }_{1 \mathrm{~g}}$ mode (a) and $\mathrm{MoS}_{2} \mathrm{E}_{2 \mathrm{~g}}{ }_{\mathrm{g}}$ mode (b) as function of excitation wavelength in different thickness of InSe/MoS heterostructures. Light emission intensity map of InSe $\mathrm{A}_{1 \mathrm{~g}}^{1}$ mode (c) and $\mathrm{MoS}_{2} \mathrm{E}_{2 \mathrm{~g}}^{1}$ mode (d) in InSe $28.1 \mathrm{~nm} / \mathrm{MoS}_{2}$ heterostructure as a function of excitation wavelength. The green and red stars in (a-b) represent the experimental data taken under $532 \mathrm{~nm}$ excitation and $638 \mathrm{~nm}$ excitation respectively. The white dashed lines in (c-d) represent the $532 \mathrm{~nm}$ and $638 \mathrm{~nm}$ excitation. 
To better quantify the Raman intensity variation and hence the efficiency of the $\operatorname{InSe} / \mathrm{MoS}_{2}$ heterostructure, we introduce a simple efficiency factor F:

$$
\mathrm{F}=\frac{\text { Raman (InSe } / \mathrm{MoS}_{2} \text { heterostructure) }}{\text { Raman (InSe or } \left.\mathrm{MoS}_{2}\right)}
$$

If $\mathrm{F}$ is larger than 1 Raman intensity is enhanced in the heterostructure and if $\mathrm{F}$ is smaller than 1 , it is diminished. In Figure 4(a-f) we use this factor to compare the calculated efficiency (curves) to measurements (data points) in fifteen InSe/ $\mathrm{MoS}_{2}$ heterostructures fabricated with our random transfer method and measured using $532 \mathrm{~nm}$ or $638 \mathrm{~nm}$ incident wavelengths. The thickness of each material in the heterostructure ranges from $5 \mathrm{~nm}$ to $70 \mathrm{~nm}$. In general there is a good agreement between our model and experimental data. Discrepancies may come from a diminished measured Raman intensity due to imperfections or contamination in the heterostructure or the isolated layer despite the care taken to avoid them. Such an error in the numerator decreases the measured $\mathrm{F}$ and in the denominator increases the measured F. Figure 4(g-i) give the calculation for a continuous variation in the layer thickness of InSe/ $\mathrm{MoS}_{2}$ and for different incident wavelengths. For example, with $532 \mathrm{~nm}$ and $638 \mathrm{~nm}$ excitation, the maximum $\mathrm{F}$ for InSe are 6.17 and 4.34, in $\operatorname{InSe}(39.1 \mathrm{~nm}) / \mathrm{MoS}_{2}(27.06 \mathrm{~nm})$ and $\operatorname{InSe}(55.25 \mathrm{~nm}) / \mathrm{MoS}_{2}$ $(32.59 \mathrm{~nm})$ heterostructures respectively. For $\mathrm{MoS}_{2}$ with $532 \mathrm{~nm}$ excitation, the maximum $\mathrm{F}$ is 4.15 , in a InSe $(34.85 \mathrm{~nm}) / \mathrm{MoS}_{2}(27.68 \mathrm{~nm})$ heterostructure. Raman intensity in the heterostructures can be enhanced up to about 6 times and attenuated very severely which indicates the importance of careful designing of the optimum geometry and configuration in a heterostructure optoelectronic device. It is worth noticing that the stacking sequence of the materials has a large effect on the Raman scattering, 
which can be seen from the Raman intensity and enhancement factor F simulation of reversed $\mathrm{MoS}_{2} / \mathrm{InSe}$ heterostructure in the supplementary information 4.
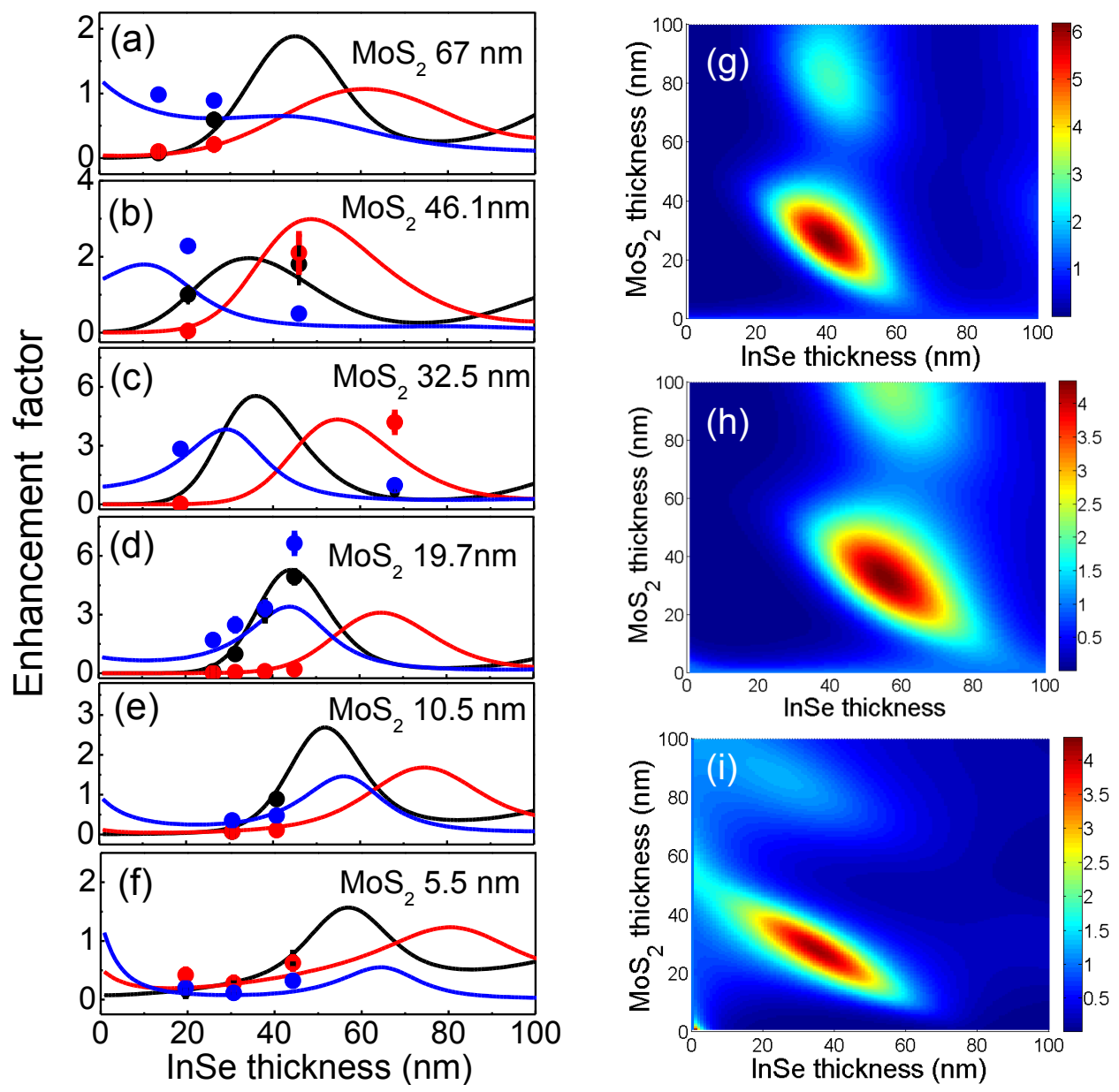

Figure 4. Raman enhancement factor $(F)$ in $I n S e / M_{0} S_{2}$ heterostructure. (a-f) Comparison of $F$ between experimental data (dots) and calculation (curves) as function of InSe thickness under different $\mathrm{MoS}_{2}$ thicknesses. The black and red curves represent the calculated F of InSe $\mathrm{A}_{1 \mathrm{~g}}{ }_{1 \mathrm{~g}}$ mode under $532 \mathrm{~nm}$ and 638 $\mathrm{nm}$ excitation respectively. The blue curve represents the calculated $\mathrm{F}$ of $\mathrm{MoS}_{2} \mathrm{E}_{2 \mathrm{~g}}^{1}$ mode under $532 \mathrm{~nm}$ excitation. (g-i) Calculated $\mathrm{F}$ as functions of $\mathrm{InSe}$ and $\mathrm{MoS}_{2}$ thicknesses, in which the $\mathrm{InSe} \mathrm{A}_{1 \mathrm{~g}}^{1}$ mode under $532 \mathrm{~nm}$ and $638 \mathrm{~nm}$ excitation are shown in $(\mathrm{g})$ and $(\mathrm{h})$ respectively. The $\mathrm{MoS}_{2} \mathrm{E}_{2 \mathrm{~g}}^{1}$ mode under $532 \mathrm{~nm}$ excitation is shown in (i).

We have thus verified the accuracy of our simulation for simultaneous incidence and emission in a heterostructure and we can use it to analyze the simpler case of electric field intensity distribution of the incident light in heterostructures. This analysis can provide a figure of merit for optoelectronic 
applications. According to our multi reflection and refraction model, the field intensity at depth $\mathrm{x}$ can be described as: $\mathrm{I}=\left|\mathrm{E}_{\mathrm{abs}}(\mathrm{x})\right|^{2}$. For the $\mathrm{InSe}(28.1 \mathrm{~nm}) / \mathrm{MoS}_{2}(33.9 \mathrm{~nm})$ heterostructure analyzed above, we calculated the intensity distributions through the heterostructure at normal incidence for several different incident wavelengths shown in Figure 5(a). As can be seen the intensity distributions are very dependent on the incident wavelength. The smallest variations are found for the longest wavelengths with respect to the heterostructure thickness. To confirm this finding we analyzed the field intensity distribution in another heterostructure InSe $(15.2 \mathrm{~nm}) / \mathrm{MoS}_{2}(44.8 \mathrm{~nm})$ (supplementary information 5), of the same 62 $\mathrm{nm}$ total thickness but with different thickness of each material. Even though overall distributions change, the above finding is confirmed. Thus large interference effects are found in the electric field intensity distribution in the heterostructures with thickness of few tens of nanometers, well below the wavelength of visible light. To confirm this we show a last set of simulated data where InSe is kept at a thickness of $50 \mathrm{~nm}$ while $\mathrm{MoS}_{2}$ is varied from $50 \mathrm{~nm}$ to $600 \mathrm{~nm}$. For incident wavelengths of $532 \mathrm{~nm}$ in Figure 5(b) it is immediately clear that while the $\operatorname{InSe}(50 \mathrm{~nm}) / \mathrm{MoS}_{2}(50 \mathrm{~nm})$ heterostructures show oscillating intensities due to interference effects, the heterostructures with $\mathrm{MoS}_{2}$ at $200 \mathrm{~nm}$ or higher thickness show a smoothly varying intensity distribution. It is thus important to know these distributions precisely for 2D heterostrucures where the material thickness is well below the incident light wavelength to be able to design efficient devices. 

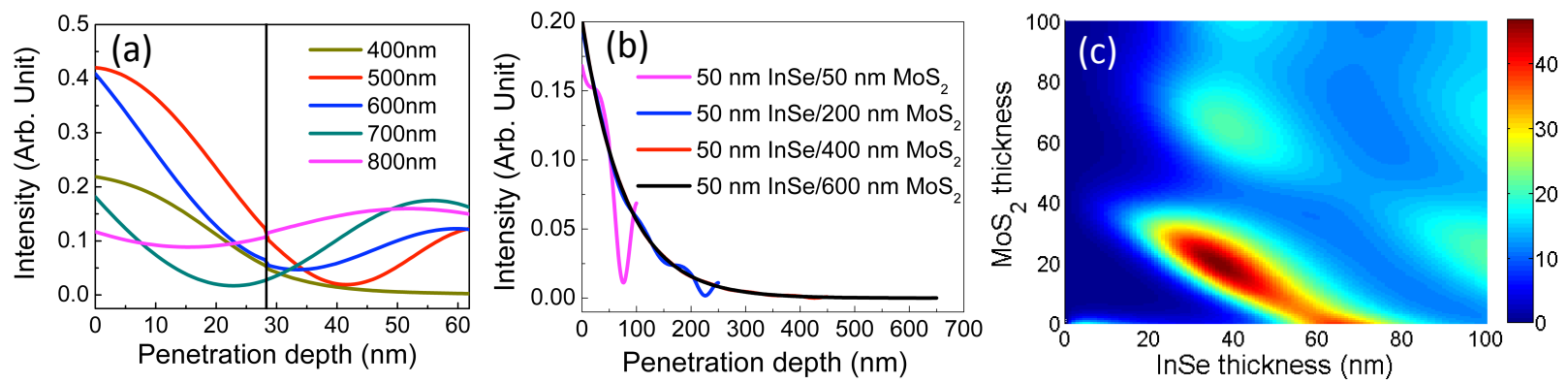

Figure 5. (a) Electric field intensity distribution in $\mathrm{InSe}(28.1 \mathrm{~nm}) / \mathrm{MoS}_{2}(33.9 \mathrm{~nm})$ heterostructure. The vertical line corresponds to the heterostructure interface. (b) Electric field intensity distribution in InSe $(50 \mathrm{~nm}) / \mathrm{MoS}_{2}$ heterostructure. (c) Intensity distribution according to formula (1) for $484 \mathrm{~nm}$ wavelength incident light (absorption maximum for $\mathrm{InSe}$ ) in a $\mathrm{InSe} / \mathrm{MoS}_{2}$ heterostructure showing an optimum for InSe $(35 \mathrm{~nm}) / \mathrm{MoS}_{2}(20 \mathrm{~nm})$.

We shortly discuss optimum absorption in a $\mathrm{InSe} / \mathrm{MoS}_{2}$ heterostructure. Figure 5(c) shows the calculated intensity according to (1) but with $\lambda_{1}=\lambda_{2}=484 \mathrm{~nm}$ (absorption maximum for InSe [40]) indicating that the optimum heterostructure is $\operatorname{InSe}(35 \mathrm{~nm}) / \mathrm{MoS}_{2}(20 \mathrm{~nm})$. However, as we show in supplementary information 6, if the stacking sequence is changed to $\mathrm{MoS}_{2} / \mathrm{InSe}$ or the substrate is changed to glass, the situation changes radically. In a device based on photocurrent measurement efficient coupling of the incident light to the device is essential. It is an important criterion in photovoltaic devices where care is taken to minimize reflection at the device surface and maximize absorption inside the device. In 2D semiconductor-heterostructures the choice of optimum thickness, stacking sequence and substrate can optimize device performance.

In conclusion, we analyzed the light interaction in $2 \mathrm{D}$ semiconductor heterostructures using $\mathrm{InSe} / \mathrm{MoS}_{2}$ as a concrete example and studying three aspects: Raman scattering intensity from the heterostructure, electric field intensity distribution inside the heterostructure and light absorption. We observed significant Raman enhancement and attenuation from experiment, and explained results precisely with our 
simulation. Both experiment and simulation results indicate that the thickness of each material, the incident wavelength and the stacking sequence have a large effect on the intensity of Raman scattering. From the intensity distribution calculations we find that in heterostructures where layer dimensions are much smaller than the incident light wavelength, tuning the 2D heterostructure geometry is necessary to optimize the performance of the device.

\section{METHODS}

$2 D$ semiconductor-heterostructure fabrication. In this article, we fabricate $2 \mathrm{D}$ semiconductorheterostructures such as $\mathrm{InSe} / \mathrm{MoS}_{2}$ and $\mathrm{GaSe} / \mathrm{InSe}$ by using our homemade random transfer method. Firstly, the bottom sample (sample 1) is prepared by mechanical exfoliation on a clean substrate (glass or $\mathrm{SiO}_{2} / \mathrm{Si}$ ) on a hot plate. The adhesion of the $2 \mathrm{D}$ precursor on the substrate is very strong when the temperature is more than $100{ }^{\circ} \mathrm{C}$ probably caused by the removal of humidity from the surface of the substrate. Since adhesive tape used for exfoliation leaves residue at high temperature we maintain a temperature in the range $100{ }^{\circ} \mathrm{C} \sim 125{ }^{\circ} \mathrm{C}$ in our fabrication process. One can take images of sample 1 after this step (image 1). Secondly, the top layer (sample 2) is then mechanically exfoliated on to heated sample $1 /$ substrate using the same procedure. After taking another images of whole samples (image 2) and compare with image 1, one can distinguish the overlapped area that cased by the second procedure, which indicate the existence of pristine sample $1 /$ sample 2 heterostructures. The detailed adhesion comparison of samples fabricated under different temperatures can be seen from supplementary information 1. 
$A F M$ and Raman characterizations. After the fabrication of 2D semiconductor-heterostructures, we characterize the surface quality and vibration modes of samples immediately to avoid any surface degradation by using AFM and Raman spectroscopy respectively. AFM scanning was performed on Scanning Probe Microscope SmartSPM-1000 instrument (AIST-NT) in ambient condition by using noncontact mode. Raman spectra and mapping were performed on a Horiba Jobin-Yvon Xplora Raman spectrometer in a back scattering geometry under ambient conditions with two kinds of incident wavelengths: $532 \mathrm{~nm}$ and $638 \mathrm{~nm}$. The focused spot of $1 \mu \mathrm{m}$ and laser power of $12 \mu \mathrm{W}$ were used to avoid lattice heating during measurement. The high yield and clean surface of 2D semiconductorheterostructure fabricated by random transfer method can be seen from supplementary information 2 .

Theoretical simulations. The detailed process of simulations can be seen in the supplementary information 3.

Conflict of Interest: The authors declare no competing financial interest.

Acknowledgement: This work was partly supported by French ANR project (Grant No. ANR-11-BS040019), by LABEX MATISSE through a post-doctoral grant and a scholarship from China Scholarship Council (CSC, Grant No. 2011618135). We acknowledge Z. Zhang for the help of calculation and A. Chevy for the precursor materials used in this work.

Supplementary Information Available: Adhesion comparison of samples fabricated under different temperatures; High yield and clean surface of 2D semiconductor-heterostructure fabricated by random transfer method; Calculation of light interaction in semiconductor-1/semiconductor- 2 heterostructure 
(light intensity distribution, light absorption and light scattering); Comparison of Raman intensity enhancement or attenuation from different stacking sequenced in 2D semiconductor-heterostructure.

\section{REFERENCES}

[1] Novoselov K S, Mishchenko A, Carvalho A and Castro Neto A H 2016 Science 82 aac9439

[2] Rivera P, Seyler K L, Yu H, Schaibley J R, Yan J, Mandrus D G, Yao W, and Xu X 2016 Science 12 688-91

[3] Withers F, Pozo-Zamudio O D, Mishchenko A, Rooney A P, Gholinia A, Watanabe K, Taniguchi T, Haigh S J, Geim A K, Tartakovskii A I and Novoselov K S 2015 Nat. Mater. 14 301-6

[4] Zhang W, Wang Q, Chen Y, Wang Z and Wee A T S 2016 2D Mater. 3022001

[5] Yu J H, Lee H R, Hong S S, Kong D, Lee H, Wang H, Xiong F, Wang S and Cui Y 2015 Nano Lett.

15, 1031-5

[6] Gong Y, Lin J, Wang X, Shi G, Lei S, Lin Z, Zou X, Ye G, Vajtai R, Yakobson B I, Terrones H, Terrones M, Tay B K, Lou J, Pantelides S T, Liu Z, Zhou W and Ajayan P M 2014 Nat. Mater. 131135 42

[7] Ross J S, Rivera P, Schaibley J, Lee-Wong E, Yu H, Taniguchi T, Watanabe K, Yan J, Mandrus D, Cobden D, Yao W and Xu X 2017 Nano Lett. 17 638-43

[8] Xu W, Liu W, Schmidt J F, Zhao W, Lu X, Raab T, Diederichs C, Gao W, Seletskiy D V and Xiong Q 2017 Nature 541, 62-7

[9] Li M Y, Chen C H, Shi Y and Li L J 2016 Mater. Today 19 322-35 
[10] He Y, Sobhani A, Lei S, Zhang Z, Gong Y, Jin Z, Zhou W, Yang Y, Zhang Y, Wang X, Yakobson

B, Vajtai R, Halas N J, Li B, Xie E and Ajayan P 2016 Adv. Mater. 28 5126-32

[11] Chen Z, Biscaras J and Shukla A 2015 Nanoscale 7 5981-6

[12] Lee C H, Lee G H, van der Zande A M, Chen W, Li Y, Han M, Cui X, Arefe G, Nuckolls C, Heinz T

F, Guo J, Hone J and Kim P 2014 Nat. Nanotechnol. 9 676-81

[13] Huo N, Kang J, Wei Z, Li S, Li J and Wei S 2014 Adv. Funct. Mater. 24 7025-31

[14] Kern J, Trügler A, Niehues I, Ewering J, Schmidt R, Schneider R, Najmaei S, George A, Zhang J, Lou J, Hohenester U, Michaelis de Vasconcellos S and Bratschitsch R 2015 ACS Photonics 2 1260-5

[15] Liu Y, Cheng R, Liao L, Zhou H, Bai J, Liu G, Liu L, Huang Y and Duan X 2011 Nat. comm. 2579

[16] Furchi M, Urich A, Pospischil A, Lilley G, Unterrainer K, Detz H, Klang P, Andrews A M, Schrenk W, Strasser G and Mueller T 2012 Nano Lett. 12 2773-7

[17] Kim M S, Roy S, Lee J, Kim B G, Kim H, Park J H, Yun S J, Han G H, Leem J Yand Kim J 2016 ACS Appl. Mater. Interfaces 8, 28809-15

[18] Bahauddin S M, Robatjazi H and Thomann I 2016 ACS Photonics 3, 853-62

[19] Lee J U, Kim K and Cheong H 2015 2D Mater. 2044003

[20] Li S L, Miyazaki H, Song H, Kuramochi H, Nakaharai S and Tsukagoshi K 2012 ACS Nano 6 73818

[21] Lien D H, Kang J S, Amani M, Chen K, Tosun M, Wang H P, Roy T, Eggleston M S, Wu M C, Dubey M, Lee S C, He J H and Javey A 2015 Nano Lett. 15 1356-61

[22] Buscema M, Steele G A, van der Zant H S J and Castellanos-Gomez A 2014 Nano Res. 7 561-71 
[23] Zhang X, Qiao X F, Shi W, Wu J B, Jiang D S and Tan P H 2015 Chem. Soc. Rev. 44 2757-85

[24] Chiu M H, Li M Y, Zhang W, Hsu W Y, Chang W H, Terrones M, Terrones H and Li L J 2014 ACS Nano 8 9649-56

[25] Wang K, Huang B, Tian M, Ceballos F, Lin M W, Mahjouri-Samani M, Boulesbaa A, Puretzky A A, Rouleau C M, Yoon M, Zhao H, Xiao K, Duscher G and Geohegan D B 2016 ACS Nano $106612-22$

[26] Chen Z, Gacem K, Boukhicha M, Biscaras J and Shukla A 2013 Nanotechnol. 24415708

[27] Dean C R, Young A F, Meric I, Lee C, Wang L, Sorgenfrei S, Watanabe K, Taniguchi T, Kim P, Shepard K L and Hone J 2010 Nat. Nanotechnol. 5 722-6

[28] Wang L, Meric I, Huang P Y, Gao Q, Gao Y, Tran H, Taniguchi T, Watanabe K, Campos L M, Muller D A, Guo J, Kim P, Hone J, Shepard K L and Dean C R 2013 Science 342 614-7

[29] Li H, Zhang Q, Yap C C R, Tay B K, Edwin T H T, Olivier, Au and Baillargeat D 2012 Adv. Funct. Mater. 22 1385-90

[30] Jin C, Kim J, Suh J, Shi Z, Chen B, Fan X, Kam M, Watanabe K, Taniguchi T, Tongay S, Zettl A, Wu J and Wang F 2017 Nature Phys. 13 127-31

[31] Wang K, Huang B, Tian M, Ceballos, Lin M, Mahjouri-Samani M, Boulesbaa A, A. Puretzky A, M. Rouleau C, Yoon M, Zhao H, Xiao K, Duscher and B. Geohegan D 2016 ACS nano $106612-22$

[32] Wang Y Y, Ni Z H, Shen Z X, Wang H M and Wu Y H 2008 Appl. Phys. Lett. 92043121

[33] Yoon D, Moon H, Son Y W, Choi J S, Park B H, Cha Y H, Kim Y D and Cheong H 2009 Phys. Rev. B 80125422 
[34] Koh Y K, Bae M H, Cahill D G and Pop E 2011 ACS Nano 5 269-74

[35] Beal A R and Hughes H P 1979 J. Phys. C: Solid State Phys. 12 881-90

[36] Grasso V and Perillo P 1977 Solid State Commun. 21 323-5

[37] Malitson I H 1965 J. Opt. Soc. Am. 55 1205-8

[38] Green M A and Keevers M J 1995 Prog. Photovolt: Res. Appl. 3 189-92

[39] Green M A 2008 Sol. Energ. Mat. Sol. Cells 92 1305-10

[40] Camassel J, Merle P, Mathieu H and Chevy A 1978 Phys. Rev. B 1, 4718-25 